\title{
Helicity, Topology and Kelvin Waves in reconnecting quantum knots
}

\author{
P. Clark di Leoni ${ }^{1}$, P.D. Mininni ${ }^{1}$, \& M.E. Brachet ${ }^{2}$ \\ ${ }^{1}$ Departamento de Física, Facultad de Ciencias Exactas y Naturales, \\ Universidad de Buenos Aires and IFIBA, CONICET, \\ Ciudad Universitaria, 1428 Buenos Aires, Argentina. \\ ${ }^{2}$ Laboratoire de Physique Statistique de l'Ecole Normale Supérieure associé au CNRS et aux Universités Paris 6 et 7, \\ 24 Rue Lhomond, 75237 Paris Cedex 05, France.
}

(Dated: February 23, 2016)

\begin{abstract}
Helicity is a topological invariant that measures the linkage and knottedness of lines, tubes and ribbons. As such, it has found myriads of applications in astrophysics and solar physics, in fluid dynamics, in atmospheric sciences, and in biology. In quantum flows, where topology-changing reconnection events are a staple, helicity appears as a key quantity to study. However, the usual definition of helicity is not well posed in quantum vortices, and its computation based on counting links and crossings of vortex lines can be downright impossible to apply in complex and turbulent scenarios. We present a new definition of helicity which overcomes these problems. With it, we show that only certain reconnection events conserve helicity. In other cases helicity can change abruptly during reconnection. Furthermore, we show that these events can also excite Kelvin waves, which slowly deplete helicity as they interact nonlinearly, thus linking the theory of vortex knots with observations of quantum turbulence.
\end{abstract}

Helicity plays an important role in the dynamics of many fluid flows. It is linked to the growth of large scale magnetic fields in astrophysics and solar physics [1], the formation of supercell convective storms in meteorology [2], the decay rate of stratified turbulence [3], and the formation of large structures in rotating and stratified flows [4] among other problems. Helicity is a measure of the knottedness of field lines, which is conserved under appropriate conditions, and as such it has been called a "topological invariant" of many flows [5]. These ideas [6-9] have found applications in areas beyond fluid dynamics, such as DNA biology [10], optics [11] and electromagnetism [12].

Although helicity is perfectly conserved in barotropic ideal fluids, in real fluids $[13,14]$ and in superfluids $[15,16]$ vortex reconnection events, which alter the topology of the flow, can take place. It is unclear how well helicity is preserved under reconnection. As a few examples, experiments of vortex knots in water have shown that center line helicity remains constant throughout reconnection events [17], while theoretical arguments indicate that writhe (one component of the helicity) should be conserved in anti-parallel reconnection events [18], a fact later confirmed in numerical simulations of a few specific quantum vortex knots [19]. However, numerical studies of Burgers-type vortices indicate that helicity is not conserved [20]. While experiments studying helicity in quantum flows have not been done yet, the recent experimental creation of quantum knots in a Bose-Einstein condensate in the laboratory [21] is a significant step in that direction.

Recently, quantum flows have been used as a testbed for many of these ideas $[17,19]$, as vorticity in a quantum flow is concentrated along vortex lines with quantized circulation, and as these vortex lines can reconnect without dissipation. However, the lack of a fluid-like definition of helicity for a quantum flow requires complex topological measurements of the linking and knottedness of vortex lines [17], or artificial filtering of the fields [19] to prevent spurious values of helicity resulting from the singularity near quantum vortices. Moreover, helicity in quantum flows has an interest per se, as reconnection events in superfluid turbulence can excite Kelvin waves [22]. These are helical perturbations that travel along the vortex lines first predicted for classical vortices by Lord Kelvin (see [23]). Kelvin waves are believed to be responsible for the generation of an energy cascade $[24,25]$ leading to Kelvin wave turbulence [26]. Possible links between helicity and the development of Kelvin wave turbulence have remain obscure as a result of the difficulties involved in the measurement of both helicity and Kelvin waves.

Here we study the time evolution of helicity and its link with Kelvin waves in numerical simulations of the GrossPitaevskii equation (GPE). The GPE models superfluids and Bose-Einstein condensates (BECs) (for which the creation of quantum knots has been recently demonstrated in the laboratory [21]) near zero temperature. We present a fluid-like regularized definition for the helicity, which solves the problem arising from the singularities in the velocity and the vorticity produced by the topological defects of the quantum flow, and links quantum knots with helicity as measured in fluid dynamics. Then, we study the time evolution of helicity in multiple linked rings and knots. We show that only some reconnection events conserve helicity, while in others helicity changes abruptly during reconnection, and later decays slowly towards a new constant value. We link this depletion of helicity to the excitation of a Kelvin wave cascade and the radiation of phonons. Finally, we illustrate how the regularized helicity can be successfully used to quantify the helicity in complicated and fully turbulent situations, such as a flow with initial large-scale helicity, and where computation of helicity by topological means would be impractical. 


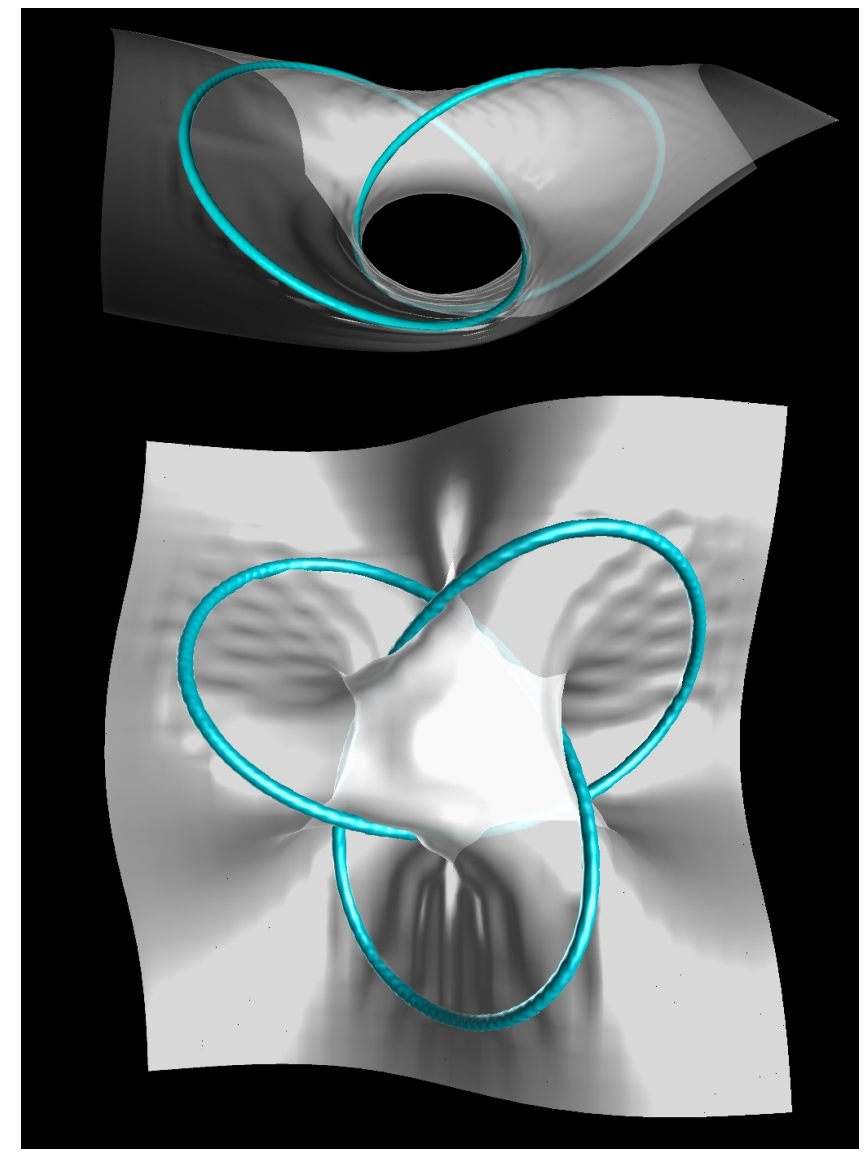

FIG. 1. Renderings of the surface of zero phase for two knots in a quantum fluid. Top: two linked rings, note the surface has one hole. Bottom: trefoil knot, with three holes. The number of holes is associated to the number of turns the vector that lies on the surface perpendicular to the vortex lines does as it moves along the curve.

Helicity in quantum flows. Low temperature quantum flows and BECs can be modeled as a field of weakly interacting bosons of mass $m$ using the GPE,

$$
i \hbar \frac{\partial \Psi}{\partial t}=-\frac{\hbar^{2}}{2 m} \nabla^{2} \Psi+g|\Psi|^{2} \Psi
$$

where $\Psi$ is the system's wavefunction and $g$ is proportional to the scattering length. The flow matches the behavior of a classical, ideal, and compressible potential fluid (i.e., it has no vorticity), except at points where a topological singularity takes place. These topological defects are the so-called quantum vortex lines where circulation is quantized and given by $\Gamma=\oint_{C} \mathbf{v}(\ell) d \ell=4 \pi \alpha$, with $\mathbf{v}$ the flow velocity and $\alpha=\hbar /(2 m)$. The vorticity $\omega$ of the flow is thus given by

$$
\boldsymbol{\omega}(\mathbf{r})=\Gamma \int \mathrm{d} s \frac{\mathrm{d} \mathbf{r}^{\prime}}{\mathrm{d} s} \delta^{(3)}\left(\mathbf{r}-\mathbf{r}^{\prime}(s)\right),
$$

where $\mathbf{r}(s)$ is the position of the vortex lines.

From the wavefunction, the particle density is given by

$$
n=\bar{\Psi} \Psi,
$$
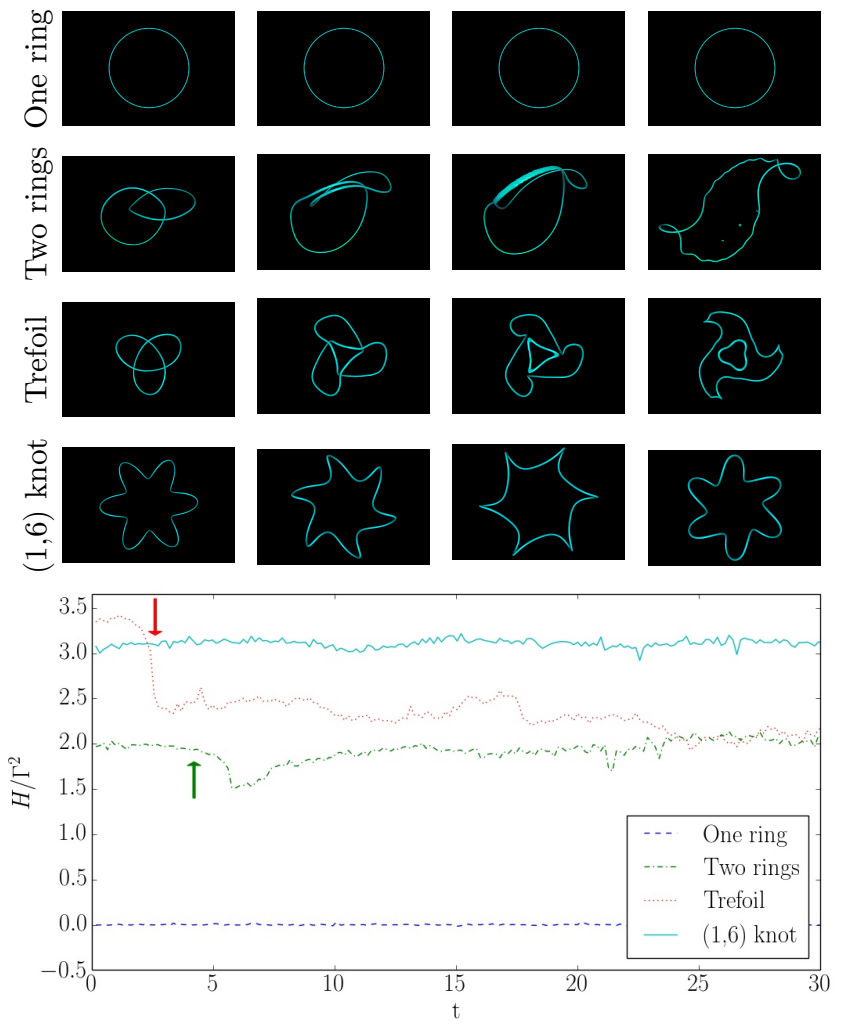

FIG. 2. Time evolution of the helicity for four quantum vortex configurations. At the top, snapshots of the configurations at different times are shown. The single ring only moves at constant speed. The two rings and the trefoil reconnect at times marked by the vertical arrows. When reconnection takes place between two anti-parallel vortex lines (as in the two rings), helicity does not change. In the trefoil reconnection takes place simultaneously at three points and helicity changes abruptly at the time indicated by the red arrow; later it decays slowly to its final value. The $(1,6)$-torus knot deforms without reconnecting, and its helicity does not change.

and the velocity field can be obtained from

$$
\mathbf{v}=\frac{\mathcal{P}}{n},
$$

where $\mathcal{P}$ is the unit mass momentum density

$$
\mathcal{P}_{j}=2 \alpha \frac{\bar{\Psi} \partial_{j} \Psi-\Psi \partial_{j} \bar{\Psi}}{2 i}
$$

(notice that these definitions are analogous to those derived via the Madelung transformation $\Psi=\sqrt{n} e^{i \phi}$, where the velocity is given by $\mathbf{v}=2 \alpha \boldsymbol{\nabla} \phi)$. At a distance $r \rightarrow 0$ from a straight vortex line these quantities are known [27] to behave as $n \sim r^{2}$ and $\mathbf{v}=2 \alpha \mathbf{e}_{\theta} / r$ where $\mathbf{e}_{\theta}$ is the azimuthal unit vector and $r$ the radial distance in a cylindrical coordinate system $\left(\mathbf{e}_{r}, \mathbf{e}_{\theta}, \mathbf{e}_{z}\right)$ having its origin on the straight vortex line. Thus, the velocity $\mathbf{v}$ has an $r^{-1}$ singularity perpendicular to the vortex line.

Therefore, as the vorticity (see Eq.(2)) also has a singularity parallel to those lines, the standard definition of 
helicity

$$
\mathcal{H}=\int \mathrm{d} \mathbf{r} \boldsymbol{\omega}(\mathbf{r}) \cdot \mathbf{v}(\mathbf{r}),
$$

is not well behaved, as it involves the product of two singular distributions. The idea of the regularized helicity is to replace in Eq. (6) the field $\mathbf{v}$ by a regularized smooth field $\mathbf{v}_{\text {reg }}$ having no divergences perpendicular to the line, and the same regular behavior as $\mathbf{v}$ parallel to the line.

Starting from Eq. (4), we can regularize the velocity along vortex lines (where $\Psi=0$ ) by Taylor expanding $\Psi$ to first order in the numerator and the denominator, arriving at

$$
v_{\|}=\frac{2 \alpha}{2 i} \frac{\left.\left.\mathcal{W}_{j}\left[\left(\partial_{j} \partial_{l} \Psi\right) \partial_{l}(\bar{\Psi})\right)-\left(\partial_{j} \partial_{l} \bar{\Psi}\right) \partial_{l}(\Psi)\right)\right]}{\sqrt{\mathcal{W}_{l} \mathcal{W}_{l}}\left(\partial_{m} \Psi\right)\left(\partial_{m} \bar{\Psi}\right)},
$$

where

$$
\mathcal{W}_{j}=\epsilon_{j k l} \partial_{k} \mathcal{P}_{l}=\frac{2 \alpha}{i} \epsilon_{j k l} \partial_{k} \bar{\Psi} \partial_{l} \Psi
$$

is a smooth field oriented along the vortex line. Then, we can define the regularized helicity

$$
\mathcal{H}=\int \mathrm{d} \mathbf{r} \boldsymbol{\omega}(\mathbf{r}) \cdot \mathbf{v}_{\mathrm{reg}}(\mathbf{r}),
$$

with $\mathbf{v}_{\text {reg }}=v_{\|} \mathcal{W} / \sqrt{\mathcal{W}_{j} \mathcal{W}_{j}}$. We show next how this regularized helicity still holds the geometrical interpretations valid for the standard one.

Relation with writhe. For an isolated structure, helicity can be decomposed into twist (loosely speaking, the total number of helical turns a ribbon does), and writhe (the "coiling" of the structure). Let's start by analyzing the relation between the regularized helicity and the writhe. For a single curve, the writhe $W r$ is, by definition [28], given by the expression

$$
W r=\frac{1}{4 \pi} \frac{\iint\left(\mathrm{d} \mathbf{r} \times \mathrm{d} \mathbf{r}_{1}\right) \cdot\left(\mathbf{r}-\mathbf{r}_{1}\right)}{\left|\left(\mathbf{r}-\mathbf{r}_{1}\right)\right|^{3}} .
$$

It is easy to see that if one uses a velocity field $\mathbf{V}(\mathbf{r})$ given by the Biot-Savart law

$$
\mathbf{V}(\mathbf{r})=\frac{\Gamma}{4 \pi} \frac{\int \mathrm{d} \mathbf{r}_{1} \times\left(\mathbf{r}-\mathbf{r}_{1}\right)}{\left|\left(\mathbf{r}-\mathbf{r}_{1}\right)\right|^{3}},
$$

where $\mathbf{r}_{1}$ corresponds to the position of the vortex lines, and the vorticity as defined in Eq. (2), then helicity $\mathcal{H}$ is given by

$$
\begin{aligned}
\mathcal{H} & =\int \mathbf{V}(\mathbf{r}) \cdot \boldsymbol{\omega}(\mathbf{r}) \mathrm{d} V=\Gamma \int \mathbf{V}(\mathbf{r}) \cdot \mathrm{d} \mathbf{r}, \\
& =\frac{\Gamma^{2}}{4 \pi} \frac{\iint \mathrm{d} \mathbf{r} \cdot\left(\mathrm{d} \mathbf{r}_{1} \times\left(\mathbf{r}-\mathbf{r}_{1}\right)\right)}{\left|\left(\mathbf{r}-\mathbf{r}_{1}\right)\right|^{3}} .
\end{aligned}
$$

From the identity $(\mathbf{a} \times \mathbf{b}) \cdot \mathbf{c}=\mathbf{a} \cdot(\mathbf{b} \times \mathbf{c})$ one finds that in this simple case (for a single line)

$$
\mathcal{H}=\Gamma^{2} W r .
$$

Regularized helicity defined as the twist of constant phase ribbon. First we recall that the twist $T w$ of a ribbon (defined by both a curve $\mathbf{r}(\mathbf{s})$, and a vector $\mathbf{U}(s)$ perpendicular to the curve) is defined by the integral over the curve

$$
T w=\frac{1}{2 \pi} \int\left(\frac{d \mathbf{U}}{\mathrm{d} s} \times \mathbf{U}\right) \cdot \frac{d \mathbf{r}}{\mathrm{d} s} \mathrm{~d} s .
$$

One can further show that $[6]$

$$
T w=N+\frac{1}{2 \pi} \int \tau(s) \mathrm{d} s,
$$

where $\tau$ is the torsion, and $N$ the number of turns round the curve of $\mathbf{U}$ in the Frenet-Serret frame (see Methods). The regularized helicity can be presented in a purely geometrical way. Under the GPE, constant phase surfaces will intersect on the vortex lines. Now consider a line at a close distance of the vortex line and lying on a constant phase surface (note that we could construct an equivalent line in the classical Biot-Savart case by requiring the line to be perpendicular to the velocity field). The vortex line and the constant phase line define a ribbon. Now, using Eqs. (2), (7) and (11) we can see that

$$
\mathcal{H}=\Gamma^{2} T w .
$$

Note that, by construction, the circulation along the constant phase line is zero.

As an illustration, Fig. 1 shows renderings of surfaces of zero phase for two knots in a quantum fluid. The presence of a hole indicates that the vector perpendicular to the vortex line lying on this surface does a whole turn as it moves along the vortex. Each of these turns contributes by one quantum $\Gamma^{2}$ to the intrinsic twist, and therefore to the helicity.

Knots in quantum flows. We consider four different initial conditions: one unknotted and unlinked ring, two unknotted but linked rings, a trefoil knot, and a $(1,6)$-torus knot (see Methods for details on the numerical scheme used, and on the preparation of the initial conditions). Snapshots at different times during their evolution are shown in Fig. 2. The single ring just moves at constant speed (parallel to the axis of axisymmetry of the ring) without any deformation. The two linked rings deform and reconnect, the reconnection taking place along segments (i.e., not at a single point) of each ring, both of them aligning anti-parallely before reconnecting. The trefoil knot undergoes three reconnections, which happen simultaneously and, in contrast with the two linked rings, do not involve long aligned segments. Finally, the $(1,6)$-torus knot deforms and moves but never reconnects. Videos showing the evolution of each configuration can be found online [29].

The evolution of the regularized helicity (normalized by $\Gamma^{2}$ ) for each configuration as a function of time is also shown in Fig. 2. A red arrow marks the time at which the trefoil reconnects, and a green arrow the moment when the two linked rings reconnect. 

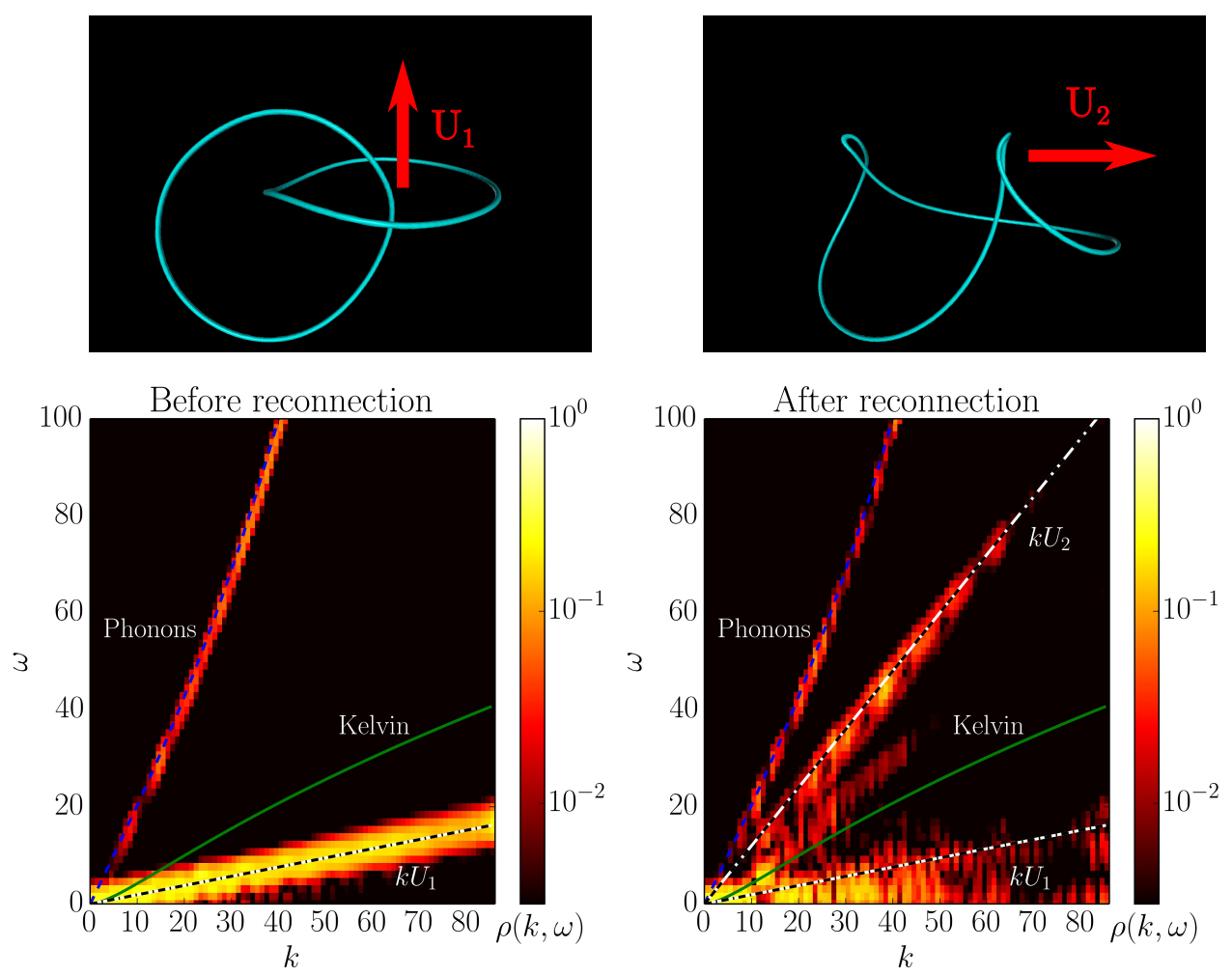

FIG. 3. Spatiotemporal spectrum for the two rings before (left) and after reconnection (right). The dashed blue line corresponds to the dispersion relation of sound waves, the solid green line to Kelvin waves, the dash-dotted line to sweeping with velocity $U_{1}$ (i.e., $\omega=U_{1} k$ ), and the dash-triple dotted line to sweeping with $\omega=U_{2} k$. Sweeping concentrates most of the power, and only one energetic mode with $k \approx 11$ may be compatible with the dispersion relation of Kelvin waves.

All four configurations start at the expected value of helicity. We verified that this value is the same as the one obtained by other methods to compute helicity in quantum flows (see, e.g., [19]). The single ring moves at constant speed without deformations, and helicity remains constant at zero. The two rings move towards each other, and align to reconnect two long anti-parallel segments (see the third pannel of the snapshots). At that time there is a small drop of the regularized helicity (associated with the fact that the regularization is not well defined while the reconnection takes place), but then the helicity remains constant around its original value of 2 , even though there remains only one ring after reconnection. This is to be expected for anti-parallel reconnection, as predicted in [18], and in agreement with previous results [17]. As is clear from the visualizations, the helicity in the link of the two rings gets converted into a helical deformation (writhe) of the single ring. The trefoil reconnects at three points simultaneously, and the vortex lines are not anti-parallel at the moment of reconnection. As a result, helicity rapidly drops by one quantum, from an initial value of $\approx 3.4$. Remarkably, it then continues dropping slowly until it reaches a new steady value of 2 quanta at $t \approx 25$. As will be shown next, this decay is associated with the excitation of helical waves along the two vortex rings resulting from the reconnection. Finally, the (1,6)-torus knot deforms substantially as it evolves, but its helicity remains around its initial value of 3 quanta.

Several conclusions can be drawn. First, there exist stable helical solutions of the GPE where vortex knots do not reconnect. Second, in agreement with previous studies $[18,19]$ antiparallel reconnections conserve helicity. Third, and in disagreement with previous claims, helicity is not conserved in all cases. Moreover, helicity can vary rapidly during reconnection, or slowly afterwards by a yet unclear mechanism. Below we show that this mechanism is the emission of phonons by the non-linear interaction of helical Kelvin-waves excited along the vortex lines.

Excitation of Kelvin waves by reconnection. To understand the process that results in the slow depletion of helicity, we compute the spatiotemporal spectrum of particle density $\rho(k, \omega)$, before and after the reconnection, for the two rings (Fig. 3) and for the trefoil (Fig. 4). This spectrum is a useful tool to identify waves and flow displacements in complex flows $[26,30]$. The GPE can sustain two types of waves that will be of interest in the following: sound waves and Kelvin waves. Sound waves follow the Bogoliubov dispersion relation $\omega_{B}(k)=c k \sqrt{1+\xi^{2} k^{2} / 2}$, where $c=\sqrt{g|\Psi|^{2} / m}$ is the speed of sound, and $\xi=\sqrt{\hbar^{2} /\left(2 m g|\Psi|^{2}\right)}$ is the coherence 

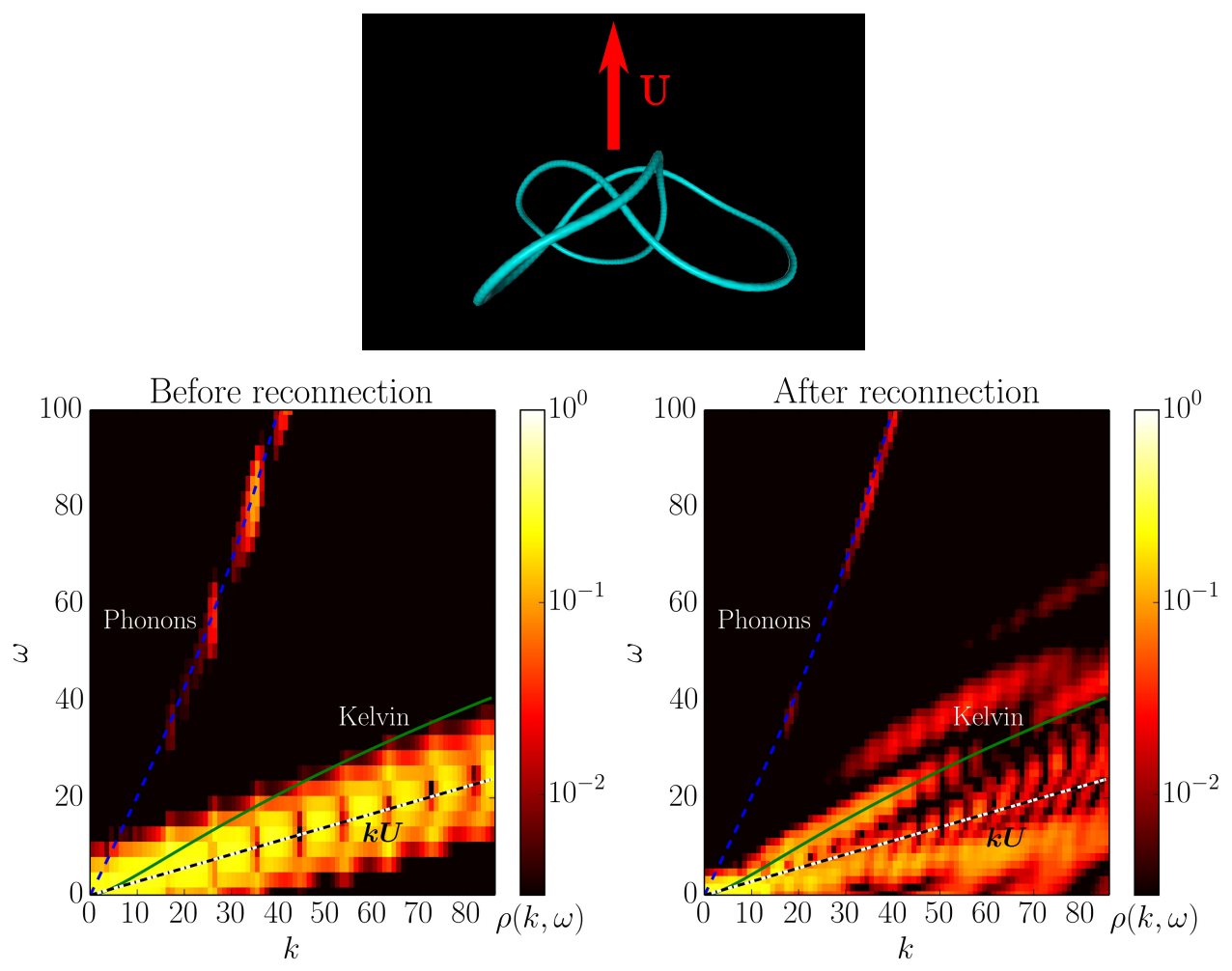

FIG. 4. Spatiotemporal spectrum for the trefoil before (left) and after the reconnection (right). The dashed blue line corresponds to sound waves, the solid green line to Kelvin waves, and the dash-dotted line to sweeping with $\omega=U k$. A broad range of modes compatible with the dispersion relation of Kelvin waves is excited after reconnection, and sound waves are visible at high frequencies.

length [27]. Kelvin waves follow the dispersion relation

$$
\omega_{K}(k)=\frac{2 c \xi}{\sqrt{2} a^{2}}\left(1 \pm \sqrt{1+\frac{K_{0}(k a)}{K_{1}(k a)}}\right),
$$

where $a$ is the radius of the vortex core, and $K_{0}$ and $K_{1}$ are modified Bessel functions.

In Fig. 3, before reconnection takes place, the two rings move towards each other at a mean velocity $U_{1}$. This appears in the spatiotemporal spectrum as sweeping of the vortices, i.e., a concentration of power near the region with $\omega=U_{1} k$ (excitations corresponding to sound waves can also be identified). After reconnection, the vortex still moves slowly with a velocity close to $U_{1}$, but the reconnected points separate fast from each other with velocity $U_{2}$. The sweeping of regions with low density (the vortex lines) associated with this velocity is also visible in the spectrum. There is almost no excitation of modes compatible with Kelvin waves, except for a single mode with $k \approx 11$ which lies on top of the dispersion relation $\omega_{K}(k=11)$. Indeed, in the last snapshot of the two rings in Fig. 2, a small helical perturbation with this wavenumber can be observed (see also the movie in the supplemental material).

The spatiotemporal spectrum for the trefoil, for which helicity is not conserved, is very different. Before reconnection the vortex knot moves with mean velocity $U$, and

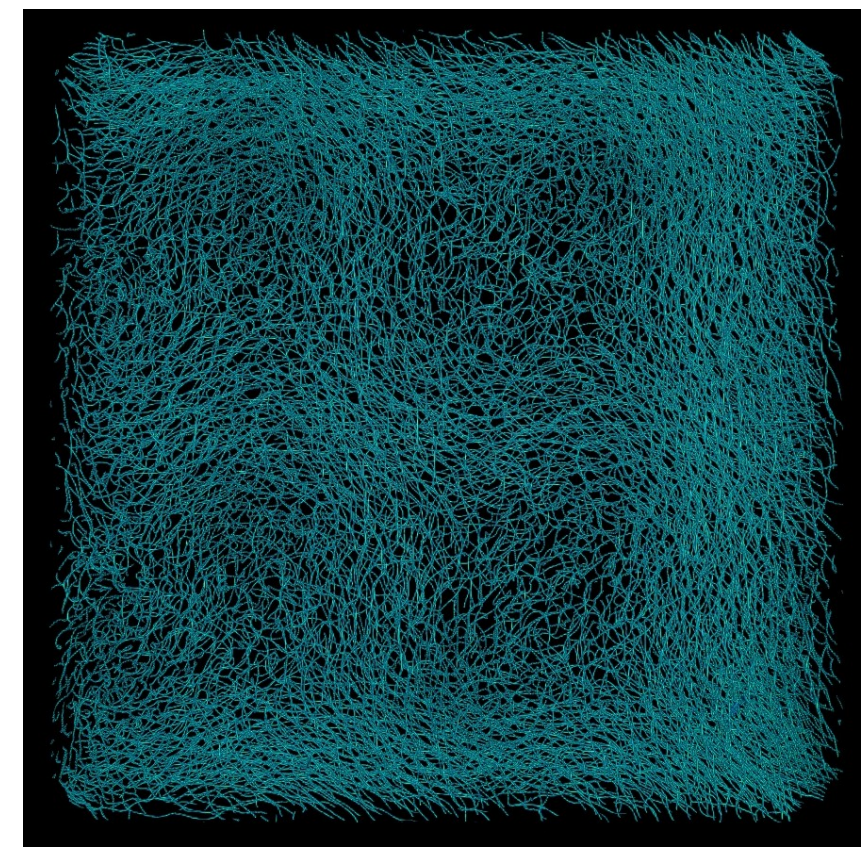

FIG. 5. Rendering of vortex lines in a quantum flow with helicity. The regularized helicity is equal to 3 , matching the value expected for the classical flow at large scales. Normalizing by the circulation quanta, the helicity of this flow is $\approx 480000 \Gamma^{2}$. 
sweeping with $\omega=U k$ can be observed in the spectrum. After reconnection the motion of the two rings is complex, although both structures still move with an average velocity $U$ (a trace can be seen in the spectrum). However, the most remarkable feature in the spectrum is the excitation of a broad range of modes compatible with the dispersion relation of Kelvin waves, and the excitation of sound waves only at high frequencies. The broad and continuous range of Kelvin wave modes indicates the development of a non-linear Kelvin wave cascade [26]: as multiple modes are excited, they can interact non-linearly and transfer their energy to larger wavenumbers, where the energy in the modes (and their helicity) can be dissipated by phonon emission [31]. Indeed, the Kelvin waves fade away once helicity reaches its new steady state value of $\approx 2$ quanta in Fig. 2 .

Helicity in complex quantum flows. Finally, we show the regularized helicity is robust even for quantum turbulence, where hundreds of thousands of knots can be present in the flow. Figure 5 shows the three-dimensional rendering of a helical flow, with a distribution of vor- tex lines such that the flow large-scale structure corresponds to the superposition of two classical ABC flows at wavenumbers $k=1$ and at $k=2$ (see Methods). The flow was computed in a grid of $2048^{3}$ grid points, with a very high density of vortex lines. Computation of the regularized helicity over the quantum flow gives the expected value of 3 , matching the classical value. In units of $\Gamma^{2}$ this value corresponds to $\approx 480000$ links.

Our observations allow the study of helicity in complex quantum knots and helical quantum turbulence, and can indicate in the future new links between helicity, Kelvin waves, and excitation of waves after reconnection, important for other areas such as fluid dynamics and space physics. The recent experimental creation of quantum knots in the laboratory [21] shows also a promising application for the quantification and evolution of the topological complexity of quantum vortices.

Acknowledgments The authors acknowledge financial support from Grant No. ECOS-Sud A13E01, and from computing hours in the CURIE supercomputer granted by Project TGCC-GENCI No. x20152a7493.
[1] A. Brandenburg and K. Subramanian, Physics Reports 417, 1 (2005).

[2] E. N. Rasmussen and D. O. Blanchard, Weather and Forecasting 13, 1148 (1998).

[3] C. Rorai, D. Rosenberg, A. Pouquet, and P. D. Mininni, Phys. Rev. E 87, 063007 (2013).

[4] R. Marino, P. D. Mininni, D. Rosenberg, and A. Pouquet, Phys. Rev. E 87 (2013), 10.1103/PhysRevE.87.033016.

[5] H. K. Moffatt, J. Fluid Mech. 35, 117 (1969).

[6] H. K. Moffatt and R. L. Ricca, Proceedings of the Royal Society of London A: Mathematical, Physical and Engineering Sciences 439, 411 (1992).

[7] M. R. Dennis and J. H. Hannay, Proceedings of the Royal Society of London A: Mathematical, Physical and Engineering Sciences 461, 3245 (2005).

[8] R. L. Ricca and M. A. Berger, Physics Today 49, 28 (2008).

[9] H. K. Moffatt, Proc. Natl. Acad. Sci. U.S.A. 111, 3663 (2014).

[10] A. V. Vologodskii, N. J. Crisona, B. Laurie, P. Pieranski, V. Katritch, J. Dubochet, and A. Stasiak, Journal of Molecular Biology 278, 1 (1998).

[11] M. R. Dennis, R. P. King, B. Jack, K. O'Holleran, and M. J. Padgett, Nature Physics 6, 118 (2010).

[12] H. Kedia, I. Bialynicki-Birula, D. Peralta-Salas, and W. T. M. Irvine, Phys. Rev. Lett. 111, 150404 (2013).

[13] F. Hussain and K. Duraisamy, Phys. Fluids 23, 021701 (2011).

[14] D. Kleckner and W. T. M. Irvine, Nature Phys. 9, 253 (2013).

[15] G. P. Bewley, M. S. Paoletti, K. R. Sreenivasan, and D. P. Lathrop, Proc. Natl. Acad. Sci. U.S.A. (2008), 10.1073/pnas.0806002105.

[16] S. Zuccher, M. Caliari, A. W. Baggaley, and C. F. Barenghi, Phys. Fluids 24, 125108 (2012).

[17] M. W. Scheeler, D. Kleckner, D. Proment, G. L. Kindl- mann, and W. T. M. Irvine, Proc. Natl. Acad. Sci. U.S.A. 111, 15350 (2014).

[18] C. E. Laing, R. L. Ricca, and D. W. L. Sumners, Scientific Reports 5, 9224 (2015).

[19] S. Zuccher and R. L. Ricca, Physical Review E 92, 061001 (2015).

[20] Y. Kimura and H. K. Moffatt, J. Fluid Mech. 751, 329 (2014).

[21] D. S. Hall, M. W. Ray, K. Tiurev, E. Ruokokoski, A. H. Gheorghe, and M. Mottonen, Nat Phys advance online publication, (2016).

[22] E. Fonda, D. P. Meichle, N. T. Ouellette, S. Hormoz, and D. P. Lathrop, Proc. Natl. Acad. Sci. U.S.A. 111, 4707 (2014).

[23] W. Thomson, Philos. Mag. 10, 155 (1880).

[24] E. Kozik and B. Svistunov, Phys. Rev. Lett. 92, 035301 (2004).

[25] V. S. L'vov and S. Nazarenko, J. Exp. Theor. Phys. Lett. 91, 428 (2010).

[26] P. Clark di Leoni, P. D. Mininni, and M. E. Brachet, Phys. Rev. A 92, 063632 (2015).

[27] C. Nore, M. Abid, and M. E. Brachet, Phys. Fluids 9, 2644 (1997).

[28] K. Klenin and J. Langowski, Biopolymers 54, 307 (2000).

[29] http://wp.df .uba.ar/mininni/movies/\#quantum.

[30] P. C. di Leoni, P. Cobelli, and P. Mininni, The European Physical Journal E 38, 1 (2015).

[31] W. Vinen, M. Tsubota, and A. Mitani, Phys. Rev. Lett. 91, 135301 (2003).

[32] D. O. Gómez, P. D. Mininni, and P. Dmitruk, Advances in Space Research 35, 899 (2005).

[33] D. O. Gómez, P. D. Mininni, and P. Dmitruk, Phys. Scripta 2005, 123 (2005).

[34] P. Mininni, D. Rosenberg, R. Reddy, and A. Pouquet, Parallel Computing 37, 316 (2011). 


\section{METHODS}

Numerical scheme. The equations were integrated using GHOST [32-34], a three dimensional code which uses a pseudospectral scheme with periodic boundary conditions to compute spatial derivatives and a fourth order Runge-Kutta scheme to compute time derivatives. The " $2 / 3$ rule" is used for de-aliasing. The code is parallelized using both MPI and OpenMP. The vortex knots simulations were done using $256^{3}$ grid points, while the ABC simulation was done with $2048^{3}$ grid points.

Preparation method for knots initial data. The initial data preparation method is based on the one presented in [17]. The method consists in calculating the velocity field generated by a vortex line (or lines) $\mathbf{r}(s)$, which is then integrated to get the phase of the wavefunction. The density at each point in space is then calculated by using a Padé approximation. One of the two differences with the method presented in [17] is that after doing this we first use the generated wavefunction as an initial condition of the advected Real Guinzbug Landau equation, whose stationary solutions are solutions of the GPE with minimal acoustic energy [27], and then feed that solutions to the GPE, thereby minimizing errors (specially those stemming from the Padé approximation). The other key difference is that our fields are truly periodic. Instead of using an array of replicas to generate an almost periodic field, we work in the Fourier domain using the Fourier transform of the vorticity (2), which as we evaluate only at integer wavenumbers gives a perfectly periodic field. The velocity field is then obtained by applying the inverse of the curl operator (i.e., the Biot-Savart law).

Preparation method for quantum ABC flow. The so-called ABC (Arnold, Beltrami and Childress) velocity field is a maximal helicity stationary solution of Euler equations in which the vorticity is parallel to the velocity, explicitly given by

$$
\begin{aligned}
\mathbf{u}_{\mathrm{ABC}}(x, y, z)=\quad & \{[B \cos (k y)+C \sin (k z)] \hat{x}+ \\
+ & {[A \sin (k x)+C \cos (k z)] \hat{y}+} \\
+ & {[A \cos (k x)+B \sin (k y)] \hat{z}\} }
\end{aligned}
$$

This velocity is the sum of three simple $(A=B=0$, $A=C=0$ and $B=C=0$ ) flows. We first construct an ARGLE initial wavefunction for each of these flows, and then take their product and run ARGLE. It is easy to see that the $A=B=0$ flow is a constant $z$-dependent advection in each $x-y$ slice. By Madelung's transformation the constant advection $C(\sin (k z) \hat{x}+\cos (k z) \hat{y})$ should correspond to a wavefunction

$$
\Psi(x, y, z)=e^{i \frac{C \sin (k z)}{2 \alpha} x+\frac{C \cos (k z)}{2 \alpha} y}
$$

In order to have a $2 \pi$-periodic initial data we initially set

$$
\Psi(x, y, z)=e^{i\left[\frac{C \sin (k z)}{2 \alpha}\right] x+\left[\frac{C \cos (k z)}{2 \alpha}\right] y}
$$

where $[a]$ stands for the integer nearer to $a$.

The general initial data is made out of a product of such functions, corresponding to non-zero $A$ and $B$ and various values of the wavenumber $k$. Note that the frustration (the relative difference between $C \sin (k z) /(2 \alpha)$ and an integer) goes down when $\alpha=c \xi / \sqrt{2} \rightarrow 0$ ).

Frenet-Serret frame and equations. We recall that, given a $3 \mathrm{D}$ curve $\mathbf{r}(s)$, with $\mathbf{r}=(x, y, z)$ and $d s=\sqrt{d x^{2}+d y^{2}+d z^{2}}$, the standard Frenet-Serret tangent $\mathbf{T}$, normal $\mathbf{N}$, and binormal $\mathbf{B}$ vectors are defined as

$$
\begin{aligned}
\frac{d \mathbf{r}}{d s} & =\mathbf{T}, \\
\frac{d \mathbf{T}}{d s} /\left\|\frac{d \mathbf{T}}{d s}\right\| & =\mathbf{N}, \\
\mathbf{T} \times \mathbf{N} & =\mathbf{B} .
\end{aligned}
$$

These obey the Frenet-Serret equations

$$
\begin{aligned}
& \frac{d \mathbf{T}}{d s}=\kappa \mathbf{N} \\
& \frac{d \mathbf{N}}{d s}=-\kappa \mathbf{T}+\tau \mathbf{B}, \\
& \frac{d \mathbf{B}}{d s}=-\tau \mathbf{N}
\end{aligned}
$$

where $\kappa$ is the curvature and $\tau$ the torsion. 